\title{
Delayed post-anoxic encephalopathy
}

\author{
AK Thacker, AB Asthana, NBS Sarkari
}

\begin{abstract}
Summary
Delayed anoxic encephalopathy, a rare yet clinically and pathologically welldelineated complication of hypoxia with unknown pathogenesis, is described in a 40-year-old man following a drug overdose.
\end{abstract}

Keywords: delayed anoxic encephalopathy, hypoxia, neurologic deficits

Neurologic deficits following acute anoxic insults are well recognised. However, severe neurologic dysfunction may be a delayed postanoxic manifestation. ${ }^{1-3}$ These are of importance due to their rare occurrence, distinctive clinical and pathologic features, and spontaneous resolution in the majority of cases. ${ }^{2}$ Most of these delayed post-anoxic neurologic sequelae have been described following carbon monoxide intoxication. The present case illustrates delayed post-anoxic encephalopathy following drug overdosage.

\section{Case report}

The patient, a 40-year-old man, was found unconscious and cyanosed due to respiratory arrest following drug overdose for attempted suicide. The exact quantity or nature of drugs taken could not be ascertained except that broken ampoules of morphine and pethidine were found at the accident site. He was resuscitated at a local hospital where he regained consciousness after $2-3$ hours. He was referred to us the next day. At the time of admission the patient was alert but withdrawn. He was oriented but had impaired recent memory. His fundii were normal and he had no focal neurologic deficit. His vital signs were stable. Blood investigations revealed haemoglobin $118 \mathrm{~g} / 1$, white cells $7.0 \times 10^{9} / 1$, erythrocyte sedementation rate $15 \mathrm{~mm}$ in first hour, glucose $5.4 \mathrm{mmol} / 1$, urea $4.4 \mathrm{mmol} / 1$, sodium $134 \mathrm{mmol} / \mathrm{l}$, potassium $3.3 \mathrm{mmol} / 1$ and normal liver function tests. Arterial blood gas analysis was normal (hydrogen ion $36 \mathrm{nmol} / 1$, bicarbonate $24 \mathrm{mmol} / 1, \mathrm{PaO}_{2} 14 \mathrm{KPa}$ and $\mathrm{PaCO}_{2}$ $4.8 \mathrm{KPa}$ ). Electro-encephalography (EEG) revealed bilateral symmetric $8-10 \mathrm{~Hz} 40 \mathrm{UV}$ alpha rhythm with no spikes, sharp complexes or slow waves. He was treated conservatively and was discharged after three days following complete recovery of his memory and emotions.

Three weeks later the patient complained of marked behavioural changes with incontinence of urine and faeces. He became withdrawn and was restless and forgetful, often making an attempt to escape from his house. He was reported to pass urine and faeces in inappropriate places such as his bed or on the sofa and expressed no guilt about this. On examination the patient had an expressionless face and his replies were often inappropriate. During examination he often stood up and it was difficult to make him sit again. His memory and orientation could not be tested. His speech was normal and cranial nerve examination including fundii did not reveal any abnormality except brisk jaw jerk. Released reflexes in the form of glabellar tap, palmo mental response, snout and grasp reflexes were prominently present. He had mild diffuse rigidity in the limbs with bilateral symmetric hyper-reflexia and extensor plantar responses. His vital signs were normal and so were his heart, chest and abdomen. His repeat investigations did not reveal any abnormality on haematologic, biochemical and cerebrospinal fluid examinations. EEG showed an abundance of $3-6 \mathrm{~Hz}$ 60-70 UV theta- and delta-waves diffusely with no spikes, sharp wave or periodic complexes. Contrast-enhanced cranial computed tomography (CT) showed bilaterally symmetric white matter diffuse low attenuation areas, mainly over the frontal region and the globus pallidus. There was no mass effect and the ventricles were of normal size. Proton magnetic resonance imaging (MRI) of the cranium in the axial plain showed evidence of bilaterally symmetric diffuse periventricular white matter hyper-intensity on $T_{1}$-weighted images predominantly in the frontal regions (figure 1).

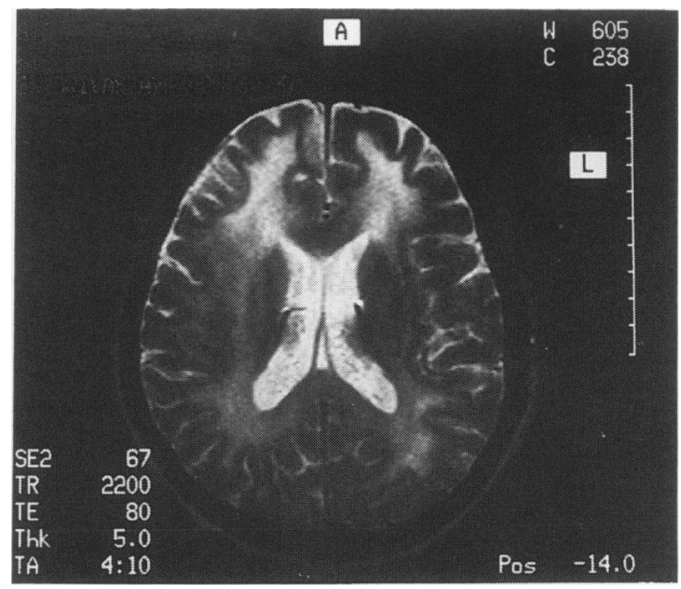

Figure 1 Predominantly frontal symmetric subcor tical white matter hyper-intensity on $\mathrm{T}_{2}$-weighted axial MRI 
Bilateral hyperintense lesions on $T_{1}$-weighted image were seen in the region of the globus pallidus (figure 2).

The patient recovered slowly during the next eight weeks without any specific therapy and could perform his duties thereafter. His clinical examination was normal. He became continent but was amnestic about his recent illness.

\section{Discussion}

After apparent complete recovery from anoxic exposure, our patient developed the symptom triad characteristic of delayed post-anoxic encephalopathy. This consists of mental deterioration, sphincteric incontinence, and gait disturbance, consequent to frontal lobe and basal ganglia dysfunctions with clinical signs of grasp reflex, glabellar tap and retropulsion due to involvement of the former and short steppage gait, masked face and rigidity due to involvement of the latter.

Delayed anoxic encephalopathy normally develops within 1-4 weeks of acute anoxic exposure, though the interval may be as short as one day to as late as $\mathbf{4 7}$ days. Unfortunately, no clinical sign or previous history distinguishes patients destined to suffer from delayed anoxic encephaloathy from those who will have an uneventful recovery. It usually affects persons in the middle or elderly age group. Most of these patients have severe anoxia with deep coma when found, but regain consciousness within 24 hours and resume full activity in 4-5 days. Many cases are mistakenly diagnosed as suffering from psychiatric disturbances. ${ }^{1}$

Delayed anoxic encephalopathy is a rare condition and has been mostly reported with carbon monoxide exposure. Shillito and Drinker ${ }^{4}$ found only 13 cases among 21000 patients with CO intoxication, while both $\mathrm{Lee}^{5}$ and $\mathrm{Choi}^{2}$ described it in $2.8 \%$ of their patients with hypoxia. The distinctive pathologic findings of extensive hemispheric subcortical demyelination and necrosis of the globus pallidus accounts for the characteristic CT and MRI findings. However, these can occur in any patient with hypoxia and are not predictors of delayed anoxic encephalopathy. ${ }^{6}$ Nevertheless, the presence of bilaterally symmetric changes in the region of the globus pallidus on CT scans indicates a poor outcome. ${ }^{7}$

Despite definitive clinical and pathologic features the exact pathogenesis of delayed anoxic encephalopathy still eludes us. Attempts to attribute striking demyelination to carbon monoxide exposure, cerebral blood

1 Plum F, Posner JB, Hain RF. Delayed neurological deterioration after anoxia. Arch Intern Med 1962; 110. 56-63.

2 Choi IS. Delayed neurologic sequelae in carbon monoxide intoxication. Arch Neurol 1983; 40: 433-5

3 Hori A, Hirose G, Kataoka S, Tsukada K, Furui K, Tonam $H$. Delayed post anoxic encephalopathy after strangulation. Serial neuroradiological and neuro chemical studies. Arch Neurol 1991; 48: 871-4.

4 Shillito FH, Drinker CK. The problem of nervous and mental sequelae in carbon monoxide poisoning. $\mathcal{F} A M A$ 1936; 106: 669-74.

5 Lee MH. Clinical studies on delayed sequelae of carbon monoxide intoxication. $\mathcal{f}$ Korean Neuropsychiatry Assoc 1978; 15: $374-85$.

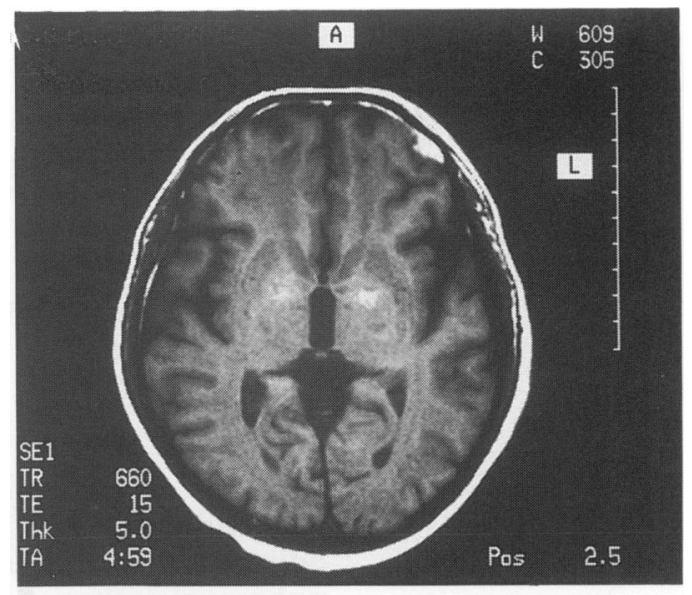

Figure $2 T_{1}$-weighted axial MRI with bilateral hyper-intensities in the globus pallidus

\section{Learning points: delayed anoxic} encephalopathy

- rare but well recognised entity usually manifesting 1-4 weeks after anoxic exposure

- characteristic subcortical white matter demyelination with necrosis of globus pallidus responsible for clinical, CT and MRI findings. However, pathogenesis uncertain

- triad of mental deterioration, sphincteric incontinence and gait disturbances with dementia, pseudo-bulbar palsy and parkinsonism on examination

- no specific therapy known but majority recover spontaneously

vessel damage, cerebral oedema, or hypersensitivity reactions, such as that found in experimental allergic encephalomyelitis, possess either serious omissions or are contradicted by extant data. ${ }^{1,2}$ The recent demonstration of pseudo-deficiency of arylsulfatase $\mathrm{A}$ in a patient with delayed anoxic encephalopathy and reversible alteration of cerebrospinal fluid $\gamma$-aminobutyric acid and dopamine concentrations following recovery in another may have implications in our understanding of its pathogenesis. ${ }^{3,8}$

There is no specific therapy or prophylaxis for delayed anoxic encephalopathy. Fortunately, its outcome is relatively good, with $50-75 \%$ of patients having full recovery as in our patient. Persisting late sequelae include memory disturbances and parkinsonism in some.

6 Ginsberg MD, Hedley-Whyte ET, Richardson EP Hypoxic-ischemic leukoencephalopathy in man. Arch Hypoxic-ischemic leuk

7 Sawada Y, Takahashi M, Ohashi H, et al. Computerised tomography as an indication of long term outcome after acute carbon monoxide poisoning. Lancet 1980; 2: 783-4 8 Weinberger LM, Schmidley JW, Schafer IA, Raghavan S. Delayed post anoxic demyelination and arylsulfatase-A pseudo deficiency. Neurology 1994; 44: 152-4. 